\title{
HIERARCHICAL STRUCTURES AND
}

\section{ASYMMETRIC STOCHASTIC PROCESSES}

\author{
ON $p$-ADICS AND ADELES
}

W. KARWOWSKI and R. VILELA MENDES

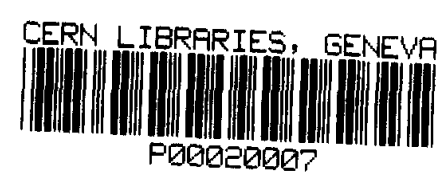

Institut des Hautes Etudes Scientifiques

35 , route de Chartres

91440 - Bures-sur-Yvette (France)

Décembre 1993

IHES/M $/ 93 / 65$ 



\title{
Hierarchical structures and asymmetric stochastic processes on p-adics and adeles
}

\author{
Witold Karwowski ${ }^{1}$ \\ Institute of Theoretical Physics, University of Wroclaw \\ Pl. Maksa Borna 9, 50-204 Wroclaw, Poland \\ R. Vilela Mendes ${ }^{2}$ \\ Institut des Hautes Etudes Scientifiques \\ 35, route de Chartres, 91440 Bures-sur-Yvette, France
}

\begin{abstract}
The space of states of some phenomena, in physics and other sciences, displays an hierarchical structure. When that is the case, it is natural to label the states by a p-adic number field. Both the classification of the states and their relationships are then based on a notion of distance with ultrametric properties. The dynamics of the phenomena, that is the transition between different states, is also a function of the p-adic distance $d_{p}$. However, because the distance is a symmetric function, probabilistic processes which depend only on $d_{p}$ have a uniform invariant probability measure, that is, all states are equally probable at large times. This being a severe limitation for cases of physical interest, we have studied processes with asymmetric transition functions. In addition to the dependence on the ultrametric distance, the asymmetric transition functions are allowed to depend also on the probability of the target state, leading to any desired invariant probability measure.

When each state of a physical system is associated to several distinct hierarchical structures or parametrizations, an appropriate labeling set is the ring of adeles. We also construct stochastic processes on the adeles.
\end{abstract}

\footnotetext{
${ }^{1}$ Supported in part by a Gulbenkian Foundation Professorship and KBN grant 4052/IFT/91

${ }^{2}$ Permanent/Mailing address: CFMC - Universidade de Lisboa; Av. Gama Pinto, 2

1699 Lisboa Codex, Portugal
} 


\section{Hierarchical structures, p-adics and adeles}

When the interactions in a complex system are such that it is not possible to optimize all the individual constraints of its elements, we have what is called a situation of frustration. The main consequence is the occurrence of a large degeneracy of the ground state and many states with energies close to the ground state. This situation occurs in disordered magnetic systems (spin glasses) ${ }^{[1-3]}$, neural networks with excitatory and inhibitory interactions and in many other optimization problems ${ }^{[4,5]}$.

One of the most interesting discoveries in these systems was the fact that the lowlying states are organized according to a tree-like hierarchical structure, with ultrametric distances between the states, that is, distances that obey the rule

$$
d(x, y) \leq \operatorname{Max}\{d(x, z), d(y, z)\}
$$

In taxonomy problems also, the notion of species is a well defined concept only if we cannot go continuously from one species to the other. Therefore the notion of ultrametric distances between species is rather natural.

If the states of a system are organized like a tree, and if the relevant dynamical distances are the ultrametric distances on the tree then, the natural number system to label the states is no longer the reals but a p-adic number field. In an infinite regular tree the states may be labeled by p-adic numbers and in a finite tree by p-adic balls. The hierarchical structures and the p-adic labeling may be used in a purely static way to characterize the equilibrium distributions at several temperatures. However, when we deal with relaxation behavior, fluctuations or transient behavior, tools must be developed to study the dynamics on p-adic spaces. Stochastic processes on trees and on p-adics have already been studied by several authors ${ }^{[6-9]}$. These authors consider the infinitesimal transition functions of the process to be functions purely of the p-adic distance. They obtain in this way a symmetric process and it is easy to see that the asymptotic probability distribution is constant over the space of states. This might be fine to study some relaxation problems but it is unrealistic if we know that the invariant probability measure is not constant in state space. This may frequently occur because, in addition to the ultrametric distance dependence, the process may have a statistical bias favoring some states more than others. This is clearly the case for example for the dynamics in the turbulent cascade tree ${ }^{[10]}$. We were therefore led to study processes with infinitesimal transition functions of the type

$$
\begin{gathered}
\tilde{\mathrm{u}}(f, i)=\rho(f) u\left(d_{f i}\right) \\
-2-
\end{gathered}
$$


where $\mathrm{u}\left(d_{f i}\right)$ is a function of the distance between the initial and the target state and $\rho(f)$ depends only on the target state. Here, by an appropriate choice of the $\rho$ functions we may obtain processes with arbitrarily chosen invariant probability measures while, at the same time, preserving the ultrametric distance-dependence of the dynamics.

If, in a complex system, each state is associated to several non-equivalent hierarchical structures then, the labeling by p-adics is no longer sufficient and the natural index set becomes the ring of adeles. This is a motivation to study also processes on the adeles. For a more detailed discussion see below.

Besides its relevance to complex systems and optimization problems, p-adics also appeared in physics coming from another field, namely string theory. In the last few years a number of papers on string theory ${ }^{[11,12]}$ studied the geometry of string worldsheets in the ultrametric case arising from p-adic norms. More recently these ideas have been related to quantum groups ${ }^{[13]}$ and cosmogenesis ${ }^{[14]}$. The result that originally caused excitement in the field was the realization that the product over all primes of the p-adic four-point functions equals the inverse of the real four-point function. This suggested some deep symmetry or, at least, that the p-adic amplitudes might be considered as building blocks of the real amplitudes. Later it was found that a similar property fails for higher order amplitudes and that even at the four-point level it depends on the way the amplitudes are extended to the p-adic fields. Nevertheless p-adic strings and, in general, p-adic quantum mechanics may still be interesting in its own right.

Many of the authors that study p-adic formulations of strings or $\mathrm{p}$-adic quantum mechanics, speculate that somehow, probably at the scale of Planck's length, other number fields besides the reals might become important for the fundamental theories of Nature. Alternatively the p-adic amplitudes are simply considered as building blocks of the real amplitudes. Concerning the use of the ring of adeles, as far as we know, Roth ${ }^{[15]}$ was the first to find an argument to justify that the adelic formulation is natural in quantum mechanics. Roth's argument is of a mathematical nature and goes as follows: In quantum mechanics we need both a description in configuration space and in momentum space. They are connected by the Fourier transform, for which we need an additive character. If one starts with the real number system in configuration space then, the most general character on the additive group $\mathbf{R}^{+}$is $\exp (\mathrm{ik} . \mathrm{x})$, where $\mathrm{k} \in \mathbf{R}$, and momentum space is also a space over the reals. However if in configuration space one starts with the rational field 
$\mathbf{Q}$, the most general additive character on $\mathbf{Q}^{+}$is

$$
\chi^{\mathbf{Q}}(x)=\exp \left[2 \pi i\left(-a_{\infty} x+a_{2} x+a_{3} x+\ldots+a_{p} x+\ldots\right)\right]
$$

where $\mathrm{p}$ runs over all primes and all but a finite number of $a_{p}$ 's are integers. Therefore momentum space becomes defined over the additive adele group $\mathbf{A}^{+}$. Thus $\mathbf{Q}^{+}$is not self-dual whereas $\mathbf{A}^{+}$is. By symmetry it is then conceivable that we should use $\mathbf{A}^{+}$in configuration space as well. The argument is of a mathematical nature and besides, it depends on an arbitrary choice of the rationals as the starting field in configuration space. Had the reals been chosen to begin with, no asymmetry would have been found between configuration and momentum spaces. Actually, as explained below, the adelic characterization of space-time appears more naturally as a consequence of general covariance considerations.

Experience shows that when looking for the laws of nature what we are likely to find are only approximate descriptions of the natural phenomena. Stable features of the natural laws cannot probably be found unless we use robust formalisms, in the sense that their features do not depend on a very accurate choice of the values of the parameters. General covariance entails such a robustness principle, in the sense that it states that the laws should not depend in the system of coordinates that is used. At a very primitive level we may think of the choice of a coordinate system as the assignment of a set of labels to a grid of points sufficiently dense to describe the phenomena we are concerned with, at the desired level of precision. Suppose now that we use an ordered set of integers to label this grid. If we have enough integers to label all the points in the grid with distinct numbers, then the labeling is finished in one round. If however the number of labels is smaller than the number of points in the grid we end up, on the average, with $\mathrm{N} / \mathrm{p}$ sites with the same label, where $\mathrm{N}$ is the total number of sites and $\mathrm{p}$ the number of distinct labels. To distinguish between the sites we are then forced to add a second label to each site and iterate the process until all sites have distinct sets of labels. In the limit of an infinite grid we therefore obtain a p-adic enumeration of the sites. To introduce a notion of distance at this level, we say that two points are close to each other if they have the same sequence of labels up to a high order. This induces exactly the topology of the p-adic distance. But does the number of different labels has to be prime? For labeling purposes nothing forbids us to choose a non-prime number q of different labels. It is as acceptable as any other for the labeling process. However, for q non-prime, a q-adic integer may always be rewritten as a p-adic integer, where $p$ is any one of the prime numbers in the primes decomposition of $q$. Therefore the non-prime labeling is not independent from the 
prime labelings. Finally, because all the prime labelings and the Z-labeling are irreducible to each other, to be fully independent of the choice of coordinates each space-time point must carry with it, as a coordinate, the full sequence of possible labels $\left(a_{\infty}, a_{2}, a_{3}, \ldots\right.$, $\left.a_{p}, \ldots\right)$.

Of course some of the properties or phenomena that take place in space-time may depend more on a particular subset of labels but, a fully universal general covariant description should be able to explore all possible labelings which therefore must be implicitly present in the space-time coordinates. To formulate continuous laws over a given subset of labels we will need to interpolate between the points of our primitive integer grid. Finally we end up with the additive group of adeles $\mathbf{A}^{+}$, where a finite number of positions belong to the field $\mathbf{Q}_{p}$ of $\mathbf{p}$-adics or to $\mathbf{R}$ and are not necessarily integers. The irreducibility of the labelings shows that dynamical structures associated to any particular $\mathrm{p}$ cannot be represented by any other $\mathrm{p}^{\prime}$ and the structures associated to the reals cannot be obtained in the limit $\mathrm{p} \rightarrow \infty$. What may happen is only that at certain scales or for certain particular. phenomena, some of the $a_{p}$ 's play a more important role than others. In all the discussion we have assumed that we end up with a labeling that distinguishes all the points in the original primitive grid. The alternative situation, where in the end some of the labels are the same, is also important, to describe topological fluctuations ${ }^{[16]}$. Notice that if labeling overlaps are allowed, they will not necessarily be the same for all $\mathrm{p}$ sectors and we may have different topology fluctuations in different p-adic sectors.

We have discussed at a somewhat longer length the motivations for adeles because they are not so well-known and because p-adics have already been discussed in many places. In Sections 2 and 3 we construct asymmetric stochastic processes on p-adics and adeles with infinitesimal transition functions of the type of Eq.(1.2). The emphasis is on explicit constructions which might actually be used in practical applications. Hence, rather than presenting our mathematical results in the lemma plus theorem plus abstract existence proof form, we have carried out a detailed step by step construction of the processes and only then, at the end, have we formalized the results as a theorem.

The time parameter, in the stochastic processes we study, is a real number. If the p-adics or the adeles are being used as a labeling set for the states of a physical system, the process represents, for example, the relaxation dynamics of the system. However, in the case discussed above of an adelic parametrization of space-time, physical time would also be an adele. In this case the real time parameter of our process should be 
considered as an additional dynamical parameter, in the same sense as the auxiliary time in stochastic quantization. The process itself should then be looked at as the establishment and fluctuation of an invariant measure in space-time.

\section{Asymmetric stochastic processes on a p-adic field}

Let $\mathrm{p}>0$ be a prime number and $\mathbf{Q}_{p}$ the field of $\mathrm{p}$-adic numbers. The set

$$
K\left(a, p^{M}\right)=\left\{x \in \mathbf{Q}_{p} ;\|a-x\|_{p} \leq p^{M}\right\}
$$

is a p-adic ball centered at $a$ with radius $p^{M}$. If the Hensel expansion of $a$ is

$$
a=\sum_{j=-m}^{\infty} \alpha_{-M+j} \quad p^{-M+j}
$$

then $K\left(a, p^{M}\right)$ is determined by the numbers

$$
\alpha_{-(M+m)}, \alpha_{-(M+m-1)}, \cdots, \alpha_{-(M+1)}
$$

which justifies the notation

$$
K\left(a, p^{M}\right) \equiv\left\{\alpha_{-(M+m)}, \cdots, \alpha_{-(M+1)}\right\}
$$

Notice that

$$
\left\{\alpha_{-(M+m)}, \cdots, \alpha_{-(M+1)}\right\}=\left\{0, \alpha_{-(M+m)}, \cdots, \alpha_{-(M+1)}\right\}
$$

and

$$
\left\{\alpha_{-(M+m)}, \cdots, \alpha_{-(M+2)}\right\}=\cup_{\alpha_{-(M+1)}^{p-1}}^{p-0}\left\{\alpha_{-(M+m)}, \cdots, \alpha_{-(M+1)}\right\}
$$

implying that, for any $M \in \mathbf{Z}, \mathbf{Q}_{p}$ is a countable union of disjoint spheres of radius $p^{M}$.

Let $\Sigma$ denote the $\sigma$-algebra generated by the set of all balls in $\mathbf{Q}_{p}$. Then, the set function defined on balls by

$$
\mu\left(K\left(a, p^{M}\right)\right)=p^{M}
$$

may be uniquely extended to a measure on $\Sigma$ which we denote by $\mu$. $\mu$ is a Haar measure for the additive group in $\mathbf{Q}_{p}$. Let $\rho$ stand for a measurable non-negative function on $\mathbf{Q}_{p}$ such that $\int_{K\left(a, p^{M}\right)} \rho(x) \mu(d x)<\infty$ for any $a \in Q_{p}$ and $M \in Z$. We set

$$
\rho_{M}^{\{\alpha\}} \equiv \rho_{\left\{\alpha_{-(M+m)}, \cdots, \alpha_{-(M+1)}\right\}}=\int_{\left\{\alpha_{-(M+m)}, \cdots, \alpha_{-(M+1)}\right\}} \rho(x) \mu(d x)=\int_{K\left(a, p^{M}\right)} \rho(x) \mu(d x)
$$


Notice that if $N \geq M+m$ then $K\left(a, p^{N}\right)=K\left(0, p^{N}\right)$ and $\rho_{N}^{\{\alpha\}}$ does not depend on $\{\alpha\}$. Finally we denote

$$
\rho_{\infty}=\int_{\mathbf{Q}_{p}} \rho(x) \mu(d x)
$$

From the physical point of view, the most interesting case is probably $\rho_{\infty}=1$. Nevertheless we will allow $\rho_{\infty}$ to take arbitrary values and if $\rho_{\infty}$ is unbounded we will simply set $\rho_{\infty}^{-1}=0$. The p-adic field $\mathbf{Q}_{\boldsymbol{p}}$ is a complete separable locally compact space with the cardinality of the continuum. However, because it is a totally disconnected space, Markov processes having this field as state space are pure jump processes. Let $\mathcal{K}^{M}=\left\{K_{i}^{M}\right\}_{i=1}^{\infty}$ be the family of disjoint balls of radius $p^{M}$ such that $\mathrm{Q}_{p}=\cup_{i=1}^{\infty} K_{i}^{M}$. We wish first to construct a stochastic process with $\mathcal{K}^{M}$ as state space. For this purpose we have to find the solutions of the forward or backward Kolmogorov equations

$$
\begin{aligned}
& \dot{P}_{K_{f} K_{i}}=-\tilde{\mathrm{a}}\left(K_{f}\right) P_{K_{f} K_{i}}(t)+\sum_{j \neq f}^{\infty} \tilde{\mathrm{u}}\left(K_{f}, K_{j}\right) P_{K_{j} K_{i}}(t) \\
& \dot{P}_{K_{j} K_{i}}=-\tilde{\mathrm{a}}\left(K_{i}\right) P_{K_{f} K_{i}}(t)+\sum_{j \neq i}^{\infty} P_{K_{f} K_{j}}(t) \tilde{\mathrm{u}}\left(K_{j}, K_{i}\right)
\end{aligned}
$$

with

$$
\tilde{\mathrm{a}}\left(K_{f}\right)=\sum_{j \neq f}^{\infty} \tilde{\mathrm{u}}\left(K_{j}, K_{f}\right)
$$

for $\mathrm{t} \geq 0$ and $\mathrm{i}, \mathrm{f} \in \mathbf{N}$, with the initial conditions $P_{K_{f} K_{i}}(0)=\delta_{i j}$.

In Refs. $[7,8]$ such a system was studied in the case where the transition kernels depend only on the p-adic distance between the balls. Here we will be interested in an asymmetric process where the transition functions depend on the target state in the manner discussed in Sect.1. Let the initial state be $K_{i}=\left\{\alpha_{-(M+m)}, \ldots, \alpha_{-(M+1)}\right\} \equiv\{\alpha\}$ and the target state be $K_{f}=\left\{\beta_{-(M+n)}, \ldots, \beta_{-(M+1)}\right\} \equiv\{\beta\}$. Notice that, by (2.3a), there is no loss of generality in assuming that $\mathrm{n}=\mathrm{m}$ and $\beta_{-(M+m)}=\alpha_{-(M+m)}$. Let $j_{0}$ be such that

$$
\beta_{-\left(M+j_{0}\right)} \neq \alpha_{-\left(M+j_{0}\right)} \text { and } \beta_{-\left(M+j_{0}+i\right)}=\alpha_{-\left(M+j_{0}+i\right)}
$$

for $\mathrm{i}=1,2, \cdots, m-j_{0}$. Then the p-adic distance of the balls is $d_{p}(\{\alpha\},\{\beta\})=p^{M+j_{0}}$. Let $\mathrm{a}(M)$ $M \in \mathbf{Z}$ be a sequence of non-negative numbers satisfying

(i) $\mathrm{a}(M) \geq \mathrm{a}(M+1)$

(ii) $\lim _{M \rightarrow \infty} \mathrm{a}(M)=0$ 
Put

$$
u(M, j)=\mathrm{a}(M+j-1)-\mathrm{a}(M+j)
$$

For the factorized transition kernels we now define

$$
\begin{gathered}
\tilde{\mathrm{u}}\left(K_{f}, K_{i}\right)=\rho_{\left\{\beta_{-(M+m)}, \ldots, \beta_{-(M+1)}\right\}} u\left(M, j_{0}\right) \\
-\tilde{\mathrm{a}}\left(K_{f}\right)=W_{\left\{\beta_{-(M+m)}, \ldots, \beta_{-(M+1)}\right\}}
\end{gathered}
$$

where $u\left(M, j_{0}\right)$ is a function that depends only on the p-adic distance of the balls and, from (2.6c), $\mathrm{W}$ is the following quantity:

$$
\begin{aligned}
& -W_{\{\beta\}}=-W_{\left\{\beta_{-(M+m)} \cdots \beta_{-(M+1)}\right\}}=u(M, 1) \sum_{\gamma_{-(M+1)} \neq \beta_{-(M+1)}} \rho_{\left\{\beta_{-(M+m)} \cdots \gamma_{-(M+1)}\right\}^{+}} \\
& u(M, 2) \sum_{\gamma_{-(M+2)} \neq \beta_{-(M+2)}} \rho_{\left\{\beta_{-(M+m)} \cdots \gamma_{-(M+2)}\right\}}+\cdots+u(M, m) \sum_{\gamma_{-(M+m)} \neq \beta_{-(M+m)}} \rho_{\left\{\gamma_{-(M+m)}\right\}}+ \\
& \sum_{j=1}^{\infty} u(M, m+j) \sum_{\gamma_{-(M+m+j)} \neq 0} \rho_{\left\{\gamma_{-(M+m+j)}\right\}}
\end{aligned}
$$

In addition, the following functions of the $\rho$ and $\mathrm{u}$ factors in the transition functions (2.8) will also be useful later on:

$$
\begin{gathered}
-\mathcal{W}_{M, j}^{\{\beta\}}=\sum_{k=j}^{m-1}(u(M, k)-u(M, k+1)) \rho_{\left\{\beta_{-(M+m)} \cdots \beta_{-(M+k+1)}\right\}}+ \\
\sum_{j=0}^{\infty}(u(M, m+j)-u(M, m+j+1)) \rho_{M+m+j}^{\{\beta\}}=\sum_{k=j}^{\infty}(u(M, k)-u(M, k+1)) \rho_{M+k}^{\{\beta\}}
\end{gathered}
$$

Notice that $\rho_{M+m+j}^{\{\beta\}}$, in the equation above, corresponds to balls with radius greater than $p^{M+m-1}$. Therefore they do not depend on $\beta$ and this index could have been dropped. The quantities $\mathcal{W}_{M, j}^{\{\beta\}}$ have the physical interpretation of time constants for jumps to a p-adic distance $p^{M+j}$. We will assume that both $W_{\{\beta\}}$ and $\mathcal{W}_{M, j}^{\{\beta\}}$ are finite quantities.

The Kolmogorov equation (2.6a) now becomes

$$
\dot{P}_{\{\beta\}\{\alpha\}}(t)=W_{\{\beta\}} P_{\{\beta\}\{\alpha\}}(t)+\rho_{\{\beta\}} \sum_{\gamma \neq \beta} u(M, j) P_{\{\gamma\}\{\alpha\}}(t)
$$

where $\mathrm{j}$ is such that $p^{M+j}=d_{p}(\{\beta\},\{\gamma\})$ and the initial condition is

$$
P_{\{\beta\}\{\alpha\}}(0)=\left\{\begin{array}{llr}
1 & \text { if } \quad\{\alpha\}=\{\beta\} \\
0 & \text { otherwise }
\end{array}\right.
$$


Let $\{\alpha\}$ be fixed. Then we may drop it to simplify notation, that is, $P_{\{\beta\}\{\alpha\}} \equiv P_{\{\beta\}}$. Denoting also

$$
P_{\left\{\gamma_{-(M+m)}, \ldots, \gamma_{-(M+j)}\right\}}=\sum_{\gamma_{-(M+1)}, \ldots, \gamma_{-(M+j-1)}=0}^{p-1} P_{\left\{\gamma_{-(M+m)}, \ldots, \gamma_{-(M+1)}\right\}}
$$

we rewrite (2.11) as follows

$$
\begin{gathered}
\dot{P}_{\left\{\beta_{-(M+m)}, \ldots, \beta_{-(M+1)}\right\}}=W_{\left\{\beta_{-(M+m)}, \ldots, \beta_{-(M+1)}\right\}} P_{\left\{\beta_{-(M+m)}, \ldots, \beta_{-(M+1)}\right\}}+\rho_{\left\{\beta_{-(M+m)}, \ldots, \beta_{-(M+1)}\right\}} \\
\left(\sum_{k=1}^{m} u(M, k) \sum_{\gamma_{-(M+k)} \neq \beta_{-(M+k)}} P_{\left\{\beta_{-(M+m), \ldots, \gamma-(M+k)}\right\}}+\sum_{i=1}^{\infty} u(M, m+i) \sum_{\gamma_{-(M+m+i)} \neq 0} P_{\left\{\gamma_{-(M+m+i)}\right\}}\right)
\end{gathered}
$$

In Eq.(2.13) each sum over $\gamma$ contains only p-1 terms. Summing and subtracting the missing terms

$$
\begin{gathered}
\left.\left.\dot{P}_{\left\{\beta_{-(M+m)}, \ldots, \beta_{-}(M+1)\right.}\right\}=\mathcal{W}_{\left\{\beta_{-}(M+m), \ldots, \beta_{-}(M+2)\right.}\right\} \\
P_{\left\{\beta_{-(M+m)}, \ldots, \beta_{-(M+1)}\right\}}+ \\
\rho_{\left\{\beta_{-(M+m), \ldots, \beta_{-}(M+1)}\right\}}\left(\sum_{k=1}^{m-1}(u(M, k)-u(M, k+1)) P_{\left\{\beta_{-(M+m)}, \ldots, \beta_{-(M+k+1)}\right.}\right\}^{+} \\
\left.\sum_{i=0}^{\infty}(u(M, m+i)-u(M, m+i+1)) P_{M+m+i}\right)
\end{gathered}
$$

where

$$
P_{M+m+i}=P_{K\left(0, p^{M+m+i}\right)}=\sum_{\beta_{-(M+m+i)}=0}^{p-1} P_{\left\{\beta_{-(M+m+i)}\right\}}
$$

Set

$$
P_{M+j}^{\{\beta\}}=P_{\left\{\beta_{-(M+m)}, \ldots, \beta_{-(M+j+1)}\right\}}
$$

Since $K\left(0, p^{M+m+i}\right)=K\left(b, p^{M+m+i}\right)$, with $b \in\left\{\beta_{-(M+m)}, \ldots, \beta_{-(M+1)}\right\}$, we may also write $P_{M+m+i}=$ $P_{M+m+i}^{\{\beta\}}$ and, with this notation, Eq.(2.14) gains a compact form, namely

$$
\dot{P}_{M}^{\{\beta\}}=\mathcal{W}_{M, 1}^{\{\beta\}} P_{M}^{\{\beta\}}+\rho_{M}^{\{\beta\}} \sum_{k=1}^{\infty}(u(M, k)-u(M, k+1)) P_{M+k}^{\{\beta\}}
$$

In all quantities in the above equation the subscript refers to the radius of the ball and the superscript to a ball of radius $p^{M}$ contained in the larger ball. Hence $P_{M+k}^{\{\rho\}}$ is the transition 
probability from the ball $\{\alpha\}$ to a ball of radius $p^{M+k}$ containing the ball $\{\beta\} . \rho_{M+k}^{\{\beta\}}$ would be the integration of the function $\rho(x)$ over the ball of radius $p^{M+k}$ that contains the ball $\{\beta\}$.

To solve Eq.(2.17) we sum successively over $\beta_{-(M+1)}, \beta_{-(M+2)}, \beta_{-(M+3)}$ and so on, to obtain a sequence of equations corresponding to an increasing sequence of concentric balls, containing the ball $\{\beta\}$. In compact notation they are

$$
\dot{P}_{M+j}^{\{\beta\}}=\mathcal{W}_{M, j+1}^{\{\beta\}} P_{M+j}^{\{\beta\}}+\rho_{M+j}^{\{\beta\}} \sum_{k=j+1}^{\infty}(u(M, k)-u(M, k+1)) P_{M+k}^{\{\beta\}}
$$

for $j \in \mathbf{N} \cup\{0\}$. The initial condition is

$$
P_{M+j}^{\{\alpha\}}(0)=1 \quad j \in \mathbf{N} \cup\{0\}
$$

If $\rho_{M+j}^{\{\beta\}}=0$ then, from $(2.18)$,

otherwise if $\rho_{M+j}^{\{\beta\}} \neq 0$

$$
P_{M+j}^{\{\beta\}}(t)=P_{M+j}^{\{\beta\}}(0) \quad e^{\imath W_{M, j+1}^{\{\beta\}}}
$$

$$
\frac{\rho_{M+j+1}^{\{\beta\}}}{\rho_{M+j}^{\{\beta\}}} \dot{P}_{M+j}^{\{\beta\}}-\dot{P}_{M+j+1}^{\{\beta\}}=\mathcal{W}_{M, j+1}^{\{\beta\}}\left(\frac{\rho_{M+j+1}^{\{\beta\}}}{\rho_{M+j}^{\{\beta\}}} P_{M+j}^{\{\beta\}}-P_{M+j+1}^{\{\beta\}}\right)
$$

For $\{\beta\}=\{\alpha\}$, using the initial condition (2.18b)

$$
\frac{\rho_{M+j+1}^{\{\alpha\}}}{\rho_{M+j}^{\{\alpha\}}} P_{M+j}^{\{\alpha\}}(t)-P_{M+j+1}^{\{\alpha\}}(t)=\left(\frac{\rho_{M+j+1}^{\{\alpha\}}}{\rho_{M+j}^{\{\alpha\}}}-1\right) e^{t \mathcal{W}_{M, j+1}^{\{\alpha\}}}
$$

Iterating the procedure we finally obtain

$$
P_{M+j}^{\{\alpha\}}(t)-\frac{\rho_{M+j}^{\{\alpha\}}}{\rho_{M+j+k}^{\{\alpha\}}} P_{M+j+k}^{\{\alpha\}}(t)=\rho_{M+j}^{\{\alpha\}} \sum_{i=0}^{k-1}\left(\frac{1}{\rho_{M+j+i}^{\{\alpha\}}}-\frac{1}{\rho_{M+j+i+1}^{\{\alpha\}}}\right) e^{t W_{M, j+i+1}^{\{\alpha\}}}
$$

Let

$$
P_{\mathbf{Q}_{p}}^{\{\alpha\}}(t)=\lim _{k \rightarrow \infty} P_{M+j+k}^{\{\alpha\}}(t)
$$

Then

$$
\begin{gathered}
P_{M+j}^{\{\alpha\}}(t)=\rho_{M+j}^{\{\alpha\}}\left\{\rho_{\infty}^{-1} P_{Q_{p}}^{\{\alpha\}}(t)+\sum_{i=0}^{\infty}\left(\frac{1}{\rho_{M+j+i}^{\{\alpha\}}}-\frac{1}{\rho_{M+j+i+1}^{\{\alpha\}}}\right) e^{t W_{M, j+i+1}^{\{\alpha\}}}\right\} \\
-10-
\end{gathered}
$$


If $\rho_{\infty}^{-1}=0$ then $P_{M+j}^{\{\alpha\}}(t)$ is uniquely determined by Eq.(2.23). If however $\rho_{\infty}^{-1} \neq 0$, we have the freedom to choose $P_{\mathbf{Q}_{\mathbf{p}}}^{\{\alpha\}}(t)$. In particular we may choose $P_{\mathbf{Q}_{p}}^{\{\alpha\}}(t)=1$.

Consider now $\{\beta\}(\neq\{\alpha\})$ to be a ball of radius $p^{M}$ at a distance $p^{M+j_{0}}$ from the ball $\{\alpha\}$. According to (2.7)

$$
\begin{aligned}
& \rho_{M+j_{0}+k}^{\{\alpha\}}=\rho_{M+j_{0}+k}^{\{\beta\}} \\
& \mathcal{W}_{M, j_{0}+k}^{\{\alpha\}}=\mathcal{W}_{M, j_{0}+k}^{\{\beta\}}
\end{aligned}
$$

for $\mathrm{k} \in \mathrm{N} \cup\{0\}$

It follows that whenever $j \geq j_{0}$ the Eqs.(2.18a) are the same for $\{\alpha\}$ or for $\{\beta\}$. Since $\left\{\beta_{-(M+m)}, \ldots, \beta_{-\left(M+j_{0}+1\right)}\right\}=\left\{\alpha_{-(M+m)}, \ldots, \alpha_{-\left(M+j_{0}+1\right)}\right\}$, the initial condition is also the same. Therefore $P_{M+j}^{\{\alpha\}}(t)=P_{M+j}^{\{\beta\}}(t)$ for $j \geq j_{0}$. Now, for $j=j_{0}-1$, Eq. (2.20) reads

$$
\frac{\rho_{M+j_{0}}^{\{\alpha\}}}{\rho_{M+j_{0}-1}^{\{\beta\}}} \dot{P}_{M+j_{0}-1}^{\{\beta\}}-\dot{P}_{M+j_{0}}^{\{\alpha\}}=\mathcal{W}_{M, j_{0}}^{\{\alpha\}}\left(\frac{\rho_{M+j_{0}}^{\{\alpha\}}}{\rho_{M+j_{0}-1}^{\{\beta\}}} P_{M+j_{0}-1}^{\{\beta\}}-P_{M+j_{0}}^{\{\alpha\}}\right)
$$

Because $\left\{\beta_{-(M+m)}, \ldots, \beta_{-\left(M+j_{0}\right)}\right\} \not \supset\left\{\alpha_{-(M+m)}, \ldots, \alpha_{-(M+1)}\right\}$ the initial condition is

$$
P_{M+j_{0}-1}^{\{\beta\}}(0)=0
$$

and

$$
P_{M+j_{0}}^{\{\alpha\}}(0)=1
$$

Integrating (2.25) with this initial condition and substituting (2.23) we obtain

$$
\begin{gathered}
P_{M+j_{0}-1}^{\{\beta\}}(t)=\rho_{M+j_{0}-1}^{\{\beta\}}\left\{\rho_{\infty}^{-1} P_{\mathbf{Q}_{p}}(t)+\right. \\
\sum_{k=0}^{\infty}\left(\frac{1}{\rho_{M+j_{0}+k}^{\{\alpha\}}}-\frac{1}{\rho_{M+j_{0}+k+1}^{\{\alpha\}}}\right) e^{\left.t \mathcal{W}_{M, j_{0}+k+1}^{\{\alpha}-\frac{1}{\rho_{M+j_{0}}^{\{\alpha\}}} e^{t \mathcal{W}_{M, j_{0}}^{\{\alpha\}}}\right\}}
\end{gathered}
$$

For $j=j_{0}-k$ with $k \geq 2$, the initial conditions imply that in

$$
\frac{\rho_{M+j_{0}-k+1}^{\{\beta\}}}{\rho_{M+j_{0}-k}^{\beta \beta\}}} P_{M+j_{0}-k}^{\{\rho\}}(t)-P_{M+j_{0}-k+1}^{\{\beta\}}(t)=C e^{t \mathcal{W}_{M, j_{0}-k+1}^{\{\beta\}}}
$$

the constant $\mathrm{C}$ is zero and therefore

$$
P_{M+j_{0}-k}^{\{\beta\}}(t)=\frac{\rho_{M+j_{0}-k}^{\{\beta\}}}{\rho_{M+j_{0}-k+1}^{\{\beta\}}} P_{M+j_{0}-k+1}^{\{\beta\}}(t)
$$


Our aim is to construct the process on balls of radius $p^{M}$, that is, to find $P_{\left\{\beta_{-(M+m)}, \ldots, \beta_{-(M+1)}\right\}}(t)$. Iterating Eq.(2.28) until one reaches the level of the balls of radius $p^{M}$ one obtains finally

$$
\begin{aligned}
& P_{\left\{\beta_{-(M+m), \ldots, \beta_{-}(M+1)}\right\}}(t)=\rho_{\left\{\beta_{-(M+m)}, \ldots, \beta_{-(M+1)}\right\}}\left\{\rho_{\infty}^{-1} P_{Q_{p}}(t)+\right. \\
& \left.\sum_{k=0}^{\infty}\left(\frac{1}{\rho_{M+j_{0}+k}^{\{\alpha\}}}-\frac{1}{\rho_{M+j_{0}+k+1}^{\{\alpha\}}}\right) e^{t \mathcal{W}_{M, j_{0}+k+1}^{\{\alpha\}}}-\frac{1}{\rho_{M+j_{0}}^{\{\alpha\}}} e^{t \mathcal{W}_{M, j_{0}}^{\{\alpha\}}}\right\}
\end{aligned}
$$

Eq.(2.29) completes the construction of the process on balls of radius $p^{M}$. All transition probabilities are obtained explicitly, the $\rho$ 's and $\mathcal{W}$ 's being, by (2.5a) and (2.10), functions of the infinitesimal transition probabilities.

If $\rho_{\infty}=1, P_{\mathbf{Q}_{p}}(\mathrm{t})=1$ and all $\mathcal{W}$ 's are negative quantities

$$
\lim _{t \rightarrow \infty} P_{\left\{\beta_{-(M+m)}, \ldots, \beta_{-(M+1)}\right\}}(t)=\rho_{\left\{\beta_{-(M+m)}, \ldots, \beta_{-(M+1)}\right\}}
$$

meaning that we may construct a process of this nature for any specified invariant probability measure. If we choose $P_{\mathbf{Q}_{p}}(\mathrm{t})$ to be a decreasing function then we have a killing of the process.

To obtain now a stochastic process on $Q_{p}$ from the process on the balls is straightforward. The process on $\mathrm{Q}_{p}$ will be defined by the transition functions $\mathrm{P}(a,\{\beta\})$ from an arbitrary point $a \in \mathrm{Q}_{p}$ to a ball $\{\beta\}$. If $\{\beta\}$ is a ball of radius $p^{M}$, all one has to do is to identify the ball of radius $p^{M}$ that contains $a$. Call it $\{\alpha\}$. And now we reinterpret Eq.(2.29) as the transition function $P(a,\{\beta\})$. For the consistency of this construction the fact that the functions $u(M, j)$ are obtained from the sequence $\mathrm{a}(\mathrm{M})$ by Eq.(2.8a) plays an important role. From (2.8a) it follows that $u(M, j)=u(M+j)$, hence also $\mathcal{W}_{M, j}^{\{\alpha\}}=\mathcal{W}_{M+j}^{\{\alpha\}}$. This insures that it is the same to consider the probability of a transition from a point to a ball of radius $p^{M}$ or to the union of the balls of radius $p^{M-1}$ contained in the larger ball. We summarize our results in the following

Theorem 2.1 - Let the infinitesimal transition functions $(2.8 \mathrm{~b})$ be such that the quantities $\mathrm{W}$ and $\mathcal{W}$ defined by Eqs.(2.9) and (2.10) are finite. Then there is a unique Markov process in $\mathbf{Q}_{p}$, solution of the Kolmogorov equation, associated to these infinitesimal transition functions. The transition functions of the process are given explicitly, for all times, by Eq.(2.29). For each set of monotonously decreasing functions $u(M+j)$ there is a process associated to any arbitrarily specified asymptotic probability distribution. 


\section{Stochastic processes on the adeles}

In $\mathbf{Q}_{p}$ the set of $\mathrm{p}$-adic integers

$$
\mathrm{Z}_{p}=\left\{x \in \mathrm{Q}_{p}:\|x\|_{p} \leq 1\right\}
$$

is a compact subgroup of the locally compact additive group in $\mathbf{Q}_{p}$. Define a restricted topological product of the p-adic fields as follows

$$
\mathbf{A}_{\mathbf{Q}}=\left\{\left(x_{p}\right) \in \prod_{p} \mathbf{Q}_{p} ; x_{p} \in \mathbf{Z}_{p} \text { for almost all } \mathrm{p}\right\}
$$

In $\mathbf{A}_{\mathbf{Q}}$ only a finite number of components are allowed not to be a p-adic integer. We topologize $\mathbf{A}_{\mathbf{Q}}$ by taking as basic open sets the products $\prod_{p} Z_{p}$, where $Z_{p}$ is an open set in $\mathbf{Q}_{p}$ and $Z_{p}=\mathbf{Z}_{p}$ for almost all $\mathrm{p}$. If $\mathbf{A}_{\mathbf{Q}}$ is endowed with componentwise operations it becomes a locally compact topological ring. $\mathbf{A}_{\mathbf{Q}}$ is called the ring of adeles .

$\mathbf{A}_{\mathbf{Q}}$ is a measurable space with Borel $\sigma$-algebra $\mathcal{F}_{\mathbf{Q}}$ generated by

$$
F_{\mathbf{Q}}=\left\{M=\prod_{p} M_{p} \text { where } M_{p} \text { is Borel in } \mathbf{Q}_{p} \text { and } M_{p}=\mathbf{Z} \text { for almost all } \mathrm{p}\right\}
$$

The measure defined in $Q_{p}$ by

$$
\mu_{p}\left(K\left(a, p^{M}\right)\right)=p^{M} \quad \mathrm{~K}\left(\mathrm{a}, p^{M}\right)=\left\{x \in \mathbf{Q}_{p} ;\|a-x\|_{p} \leq p^{M}\right\}
$$

is a Haar measure on the locally compact additive group in $\mathbf{Q}_{p}$. Notice that $\mu_{p}\left(\mathbf{Z}_{p}\right)=1$. Then, for $M \in F_{\mathbf{Q}}$ we set

$$
\mu(M)=\prod_{p} \mu_{p}\left(M_{p}\right)
$$

The set function $\mu$ may be uniquely extended to the $\sigma$-algebra $\mathcal{F}_{\mathbf{Q}}$. The extension, also denoted by $\mu$, is the Haar measure on the locally compact additive group on $\mathbf{A}_{\mathbf{Q}}$. Uniqueness of the extension follows from the fact that $F_{\mathbf{Q}}$ is closed under finite intersections and, if $B_{n}$ is the set

$$
B_{n}=K\left(a, p_{1}^{n}\right) \times K\left(a, p_{2}^{n-1}\right) \times \cdots \times K\left(a, p_{n}^{0}\right) \times \mathbf{Z}_{p_{n+1}} \times \cdots
$$

then $\mu\left(B_{n}\right)<\infty$ and $\mathbf{A}_{\mathbf{Q}}=\cup_{n} B_{n}$. Notice that $B_{n}$ is compact for all $\mathrm{n} \in \mathbf{N}$ and since $\mathbf{A}_{\mathbf{Q}}=\cup_{n} B_{n}$ we conclude that $\mathbf{A}_{\mathbf{Q}}$ is $\sigma$-compact.

We are now going to construct stochastic processes on $\mathbf{A}_{\mathbf{Q}}$ using the stochastic processes in $\mathbf{Q}_{p}$ constructed in Sect.2. There we saw that, for any prime number $\mathrm{p}$, given a set of infinitesimal transition functions satisfying the conditions stated in theorem 2.1, 
there is a Markov process with transition functions $P_{p}(a,\{\beta\})$ given by Eq.(2.29), which we now rewrite emphasizing the point dependence of the initial state and labeling all quantities with the prime $\mathrm{p}$.

$$
\begin{aligned}
& P_{p}\left(a_{p},\{\beta\}_{p}\right)(t)=\rho_{M}^{\{\beta\}_{p}}\left\{\rho_{p, \infty}^{-1} P_{\mathbf{Q}_{p}}(t)+\sum_{k=0}^{\infty}\left(\left(\rho_{M+j_{0}+k}^{\{\alpha\}_{p}}\right)^{-1}-\left(\rho_{M+j_{0}+k+1}^{\{\alpha\}_{p}}\right)^{-1}\right) e^{t \mathcal{W}_{M+j_{0}+k+1}^{\{\alpha}-}\right. \\
& \left.\left(\rho_{M+j_{0}}^{\{\alpha\}_{p}}\right)^{-1} e^{t \mathcal{W}_{M+j_{0}}^{\{\alpha\}}}\right\}
\end{aligned}
$$

Recall that $\{\beta\}_{p}$ is a $\mathrm{p}$-adic ball of radius $p^{M}$ and $\{\alpha\}_{p}$ is the p-adic ball of radius $p^{M}$ that contains the point $a$. For the definition of the quantities $\rho$ and $\mathcal{W}$ and their relation to the infinitesimal transition probabilities see (2.5),(2.8) and (2.10).

Let now $B \in F_{\mathbf{Q}}, a \in \mathbf{A}_{\mathbf{Q}}, \mathrm{t} \geq 0$ with $B=\left(\{\beta\}_{p}\right) \equiv \prod_{p}\{\beta\}_{p}$ and $a=\left(a_{p}\right) \equiv \prod_{p} a_{p}$. We define the following transition function in $\mathbf{A}_{\mathbf{Q}}$

$$
P(a, B)(t)=\prod_{p} P_{p}\left(a_{p},\{\beta\}_{p}\right)(t)
$$

We are using the stochastic processes in $\mathbf{Q}_{p}$ that were constructed in Sect. 2 to induce in a componentwise way a stochastic process in $\mathbf{A}_{\mathbf{Q}}$. However there are two things that have to be checked. First, because $\mathbf{A}_{\mathbf{Q}}$ is the restricted topological product (3.2) we have to check that, under time evolution, the process indeed remains in $\mathbf{A}_{\mathbf{Q}}$ with probability one. Second we should check that the product of the transition functions of each one of the $\mathbf{Q}_{p}$-processes defines a transition function in the whole space $\mathbf{A}_{\mathbf{Q}}$.

\# Sufficient conditions for the process to stay in $\mathbf{A}_{\mathbf{Q}}$ with probability one

We require

$$
\prod_{p}^{\infty} P_{p}\left(x_{p}, \mathbf{z}_{p}\right)(t)>0
$$

for $x_{p} \in \mathbf{Z}_{p}$ and $0 \leq t<T, T$ being the time until when the process is defined. $\mathbf{Z}_{p}$ is a $p$-adic ball of radius 1 and $P_{p}\left(x_{p}, Z_{p}\right)$ is the probability to remain inside this ball starting from an arbitrary initial p-adic integer $x_{p}$. The condition (3.9) insures that no infinite sequence of components leaves the $\mathbf{Z}_{p}$ 's. To see this suppose that at time $0 \leq \tau<T$ the process leaves the $\mathbf{Z}_{p}$ 's for a sequence of components $p_{i_{k}}$, with positive probability. Then

$$
\begin{gathered}
\prod_{\boldsymbol{i}_{k}} P_{p_{i_{k}}}\left(\mathbf{Z}_{p_{i_{k}}}, \mathbf{Q}_{p_{i_{k}}} \backslash \mathbf{Z}_{p_{i_{k}}}\right)(\tau)>0 \\
-14-
\end{gathered}
$$


and this implies

$$
\lim _{i_{k} \rightarrow \infty} P_{p_{i_{k}}}\left(\mathbf{Z}_{p_{i_{k}}}, \mathbf{Q}_{p_{i_{k}}} \backslash \mathbf{Z}_{p_{i_{k}}}\right)(\tau) \rightarrow 1
$$

Hence

$$
\lim _{i_{k} \rightarrow \infty} P_{p_{i_{k}}}\left(\mathbf{Z}_{p_{i_{k}}}, \mathbf{Z}_{p_{i_{k}}}\right)(\tau) \rightarrow 0
$$

and

$$
\prod_{p}^{\infty} P_{p}\left(x_{p}, \mathbf{z}_{p}\right)(\tau)=0
$$

From Eq.(2.23) it follows

$$
P_{p}\left(x_{p}, \mathbf{Z}_{p}\right)(t)=P_{0}^{\mathbf{Z}_{p}}(t)=\rho_{0}^{\mathbf{Z}_{p}}\left\{\rho_{p, \infty}^{-1} P_{\mathbf{Q}_{p}}(t)+\sum_{k=0}^{\infty}\left(\frac{1}{\rho_{k}^{\mathbf{Z}_{p}}}-\frac{1}{\rho_{k+1}^{\mathbf{Z}_{p}}}\right) e^{t \boldsymbol{W}_{o, k+1}^{\mathbf{z}_{p}}}\right\}
$$

Eq.(3.9) will be satisfied if the infinitesimal transition functions are chosen, for each p, in such a way that the infinite product does not vanish. It is however useful to derive sufficient conditions that are easily checked and insure that (3.9) holds for all times.

(a) Let $\rho_{p_{1} \infty}^{-1}=1$ and $P_{\mathbf{Q}_{p}}(t)=1 \forall$ p. Then $P_{0}^{\mathbf{Z}_{p}}(t) \geq \rho_{0}^{\mathbf{Z}_{p}}$ and a sufficient condition for (3.9) to hold is

$$
\prod_{p} \rho_{0}^{Z_{p}}>0
$$

(b) Let now $\rho_{p, \infty}^{-1}=0$. Then $P_{0}^{z_{p}}(t) \geq \rho_{0}^{\mathbf{z}_{p}}\left(\left(\rho_{0}^{\mathbf{z}_{p}}\right)^{-1}-\left(\rho_{1}^{z_{p}}\right)^{-1}\right) e^{t \boldsymbol{w}_{0,1}^{\mathbf{z}_{p}}}$ and a sufficient condition for $(3.9)$ is

$$
\prod_{p}\left(1-\frac{\rho_{0}^{\mathbf{z}_{p}}}{\rho_{1}^{\mathbf{z}_{p}}}\right)>0 \text { and }-\sum_{p} \mathcal{W}_{0,1}^{\mathbf{z}_{p}}<\infty
$$

Now we have to check that $P(a, B)(t)$ is indeed the transition function of a Markov process. The set function $P(a, \cdot)(t)$ may be uniquely extended to the $\sigma$-algebra $\mathcal{F}_{\mathbf{Q}}$. \#P(a,.) is a probability measure

To check that $P\left(a, \mathbf{A}_{\mathbf{Q}}\right)(t)=1$ notice that $\mathbf{A}_{\mathbf{Q}}=\cup_{i=1}^{\infty} \mathcal{A}_{i}$ with

$$
\begin{gathered}
\mathcal{A}_{i}=\mathbf{Q}_{p_{1}} \times \cdots \times \mathbf{Q}_{p_{i}} \times \mathbf{Z}_{p_{i+1}} \times \cdots \\
-15-
\end{gathered}
$$


Set $\mathcal{C}_{1}=\mathcal{A}_{1}$ and $\mathcal{C}_{i}=\mathcal{A}_{i+1} \backslash \mathcal{A}_{i}$ for $i>1$. We have $\mathcal{C}_{i} \cap \mathcal{C}_{j}=\emptyset$ for $i \neq j$ and $\mathcal{A}=\cup_{i=1}^{k} \mathcal{C}_{i}$. Thus $\mathbf{A}_{\mathbf{Q}}=\cup_{i=1}^{\infty} \mathcal{C}_{i}$ and $P\left(a, \mathbf{A}_{\mathbf{Q}}\right)=\sum_{i=1}^{\infty} P\left(a, \mathcal{C}_{i}\right)$. But $\sum_{i=1}^{k} P\left(a, \mathcal{C}_{i}\right)=P\left(a, \mathcal{A}_{k}\right)=\prod_{i=k+1}^{\infty} P_{p_{i}}\left(a_{p_{i}}, \mathbf{Z}_{p_{i}}\right)$ and by (3.9) this last quantity must tend to one when $\mathrm{k} \rightarrow \infty$.

$\# P(\cdot, A)$ is a measurable function for $A \in \mathcal{F}_{\mathbf{Q}}$

$P_{p}\left(x_{p}, \mathbf{Z}_{p}\right)$ remains constant when $x_{p}$ varies over $\mathbf{Z}_{p}$. If $\mathrm{A} \in F_{\mathbf{Q}}$ then, for some $\mathrm{k}>0$

$$
A=A_{p_{1}} \times \cdots \times A_{p_{k-1}} \times \mathbf{Z}_{p_{k}} \times \cdots
$$

Thus the function $P(x, A)$ restricted to

$$
\mathbf{Q}_{p_{1}} \times \cdots \times \mathbf{Q}_{p_{j}} \times \mathbf{Z}_{p_{j+1}} \times \cdots
$$

with $\mathrm{j}>\mathrm{k}$, is a direct product of $\mathrm{j}+1$ measurable functions and therefore is measurable. Let now $A, \Lambda \in F_{\mathbf{Q}}$ and put

$$
P(\Lambda, A)=\int_{\Lambda} P(x, A) \mu(d x)
$$

Eq.(3.13) defines an additive functional on $F_{\mathbf{Q}} \times F_{\mathbf{Q}}$. Because of the properties of the $F_{\mathbf{Q}}$ set the functional may be uniquely extended to a measure in $\mathbf{A}_{\mathbf{Q}} \times \mathbf{A}_{\mathbf{Q}}$. Let $\Lambda \in \mathcal{F}_{\mathbf{Q}}$ and $\mu(\Lambda)=0$. Then $P(\Lambda, A)=0$ for all $A \in F_{\mathbf{Q}}$ and, by the uniqueness of the extension, also for all $A \in \mathcal{F}_{\mathbf{Q}}$. Hence, for any fixed $A \in \mathcal{F}_{\mathbf{Q}}$, the measure $P(\Lambda, A)$ is absolutely continuous with respect to $\mu$. Hence the Radon-Nikodym derivative

$$
\frac{d P(\cdot, A)}{d \mu}=P(x, A)
$$

is a measurable function.

\# $P(x, A)$ obeys the Chapman-Kolmogorov equation

Let $q_{k}=\mathbf{Q}_{p_{1}} \times \cdots \times \mathbf{Q}_{p_{k}} ; z_{k}=\mathbf{Z}_{p_{k+1}} \times \cdots$ and $\tau_{k}=q_{k} \times z_{k}$

Now for $s, t \geq 0$ and $A \in F_{\mathbf{Q}}$ there is a sufficiently large $k$ such that

$$
\begin{aligned}
\int_{\tau_{k}} P(x, d y)(t) P(y, A)(s) & =\prod_{i=1}^{k} \int_{\mathbf{Q}_{p_{i}}} P\left(x_{i}, d y_{i}\right)(t) P\left(y_{i}, A_{i}\right)(s) \prod_{j=k+1}^{\infty} \int_{\mathbf{z}_{p_{j}}} P\left(x_{j}, d y_{j}\right)(t) P\left(y_{j}, \mathbf{z}_{p_{j}}\right)(s) \\
= & \prod_{i=1}^{k} P\left(x_{i}, A_{i}\right)(t+s) \prod_{j=k+1}^{\infty} P\left(x_{j}, \mathbf{Z}_{p_{j}}\right)(t) P\left(x_{j}, \mathbf{z}_{p_{j}}\right)(s)
\end{aligned}
$$

with $x_{j} \in \mathbf{Z}_{p_{j}}$ for $j \geq k+1$. We have $\mathbf{A}_{\mathbf{Q}}=\cup_{i=1}^{\infty} \tau_{i}$ and

$$
-16-
$$




$$
\begin{aligned}
& \lim _{k \rightarrow \infty} \prod_{i=1}^{k} P\left(x_{i}, A_{i}\right)(t+s)=P(x, A)(t+s) \\
& \lim _{k \rightarrow \infty} \prod_{j=k+1}^{\infty} P\left(x_{j}, \mathbf{Z}_{p_{j}}\right)(t) P\left(x_{j}, \mathbf{Z}_{p_{j}}\right)(s)=1
\end{aligned}
$$

Thus

$$
\int_{\mathrm{A}_{Q}} P(x, d y)(t) P(y, A)(s)=P(x, A)(t+s)
$$

By the uniqueness of the extension this relation holds also for $A \in \mathcal{F}_{\mathbf{Q}}$. Summarizing:

Theorem 3.1 Let $\tilde{u}_{p}\left(K_{f}, K_{j}\right)=\rho_{p,\{\beta\}} u_{p}\left(M, j_{0}\right)$, for all prime numbers $\mathrm{p}$, be a choice of infinitesimal transition functions satisfying the conditions of theorem 2.1. Furthermore let one of the sufficient conditions (3.11) or (3.12) be satisfied. Then the transition function given by (3.7) and (3.8) describes a Markov process on the adeles.

\section{References}

[1] M. Mezard, G. Parisi and V. Virasoro; "Spin Glass Theory and Beyond", World Scientific, Singapore 1987.

[2] R. Rammal, G. Toulouse and M. A. Virasoro; Rev. Mod. Phys. 58 (1986) 765.

[3] V. S. Dotsenko; Physics Uspekhi 36 (1993) 455.

[4] G. Parisi; in "Perspectives on Biological Complexity", page 77, O. T. Solbrig and G.

N. Nicolis (eds.) IUBS 1991.

[5] H. Epstein and D. Ruelle; Physics Reports 184 (1989) 289.

[6] A. T. Ogielski and D. L. Stein; Phys. Rev. Lett. 55 (1985) 1634.

[7] S. Albeverio and W. Karwowski; "Diffusion on p-adic numbers", Proceedings of Nagoya Conference, ed. T. Hida, 1991.

[8] S. Albeverio and W. Karwowski; "A random walk on p-adics - The generator and its spectrum", Bochum preprint SFB 237/no. 135 (1991).

[9] L. Brekke and M. Olson; " $p$-adic diffusion in glasses", Chicago preprint EFI-89-23 (1989).

[10] R. Lima and R. Vilela Mendes; "A stochastic process for the dynamics of the turbulent cascade", Marseille preprint CPT-93/P.2965.

[11] B. Grossman, Phys. Lett. B197 (1987) 101; 
P. G. O. Freund and M. Olson, Phys. Lett. B199 (1987) 186;

P. G. O. Freund and E. Witten, Phys. Lett. B199 (1987) 191;

I. V. Volovich, Class. Quant. Grav. 4 (1987) L83;

L. Brekke, P. G. O. Freund, M. Olson and E. Witten, Nucl. Phys. B302 (1988) 365;

B. L. Spokoiny, Phys. Lett. B208 (1988) 401;

P. H. Frampton and Y. Okada, Phys. Rev. Lett. 60 (1988) 484; Phys. Rev. D37 (1988) 3077 ;

Z. Hlousek and D. Spector, Phys. Lett. B214 (1988) 19;

R. B. Zhang, Phys. Lett. B209 (1988) 229;

I. Ya. Aref'eva, B. G. Dragovitch and I. V. Volovich, Phys. Lett. B209 (1988) 445; B214 (1988) 339;

L. Brekke, P. G. O. Freund, E. Melzer and M. Olson, Phys. Lett. B216 (1989) 53;

A. V. Zabrodin, Commun. Math. Phys. 123 (1989) 463.

[12] L. Brekke and P. G. O. Freund; Physics Reports 233 (1993) 1.

[13] I. Ya. Aref'eva and I. V. Volovich, Phys. Lett. B268 (1991) 179;

P. G. O. Freund, Chicago preprint EFI-90-90.

[14] P. H. Frampton and I. V. Volovich, Mod. Phys. Lett. A5 (1990) 1825;

I. Ya. Aref'eva and P. H. Frampton, Mod. Phys. Lett. A6 (1991) 313.

[15] B. D. B. Roth, Phys. Lett. B213 (1988) 263.

[16] J. M. Alberty, Phys. Lett. B221 (1989) 250; B228 (1989) 483. 
